\title{
Improving the Accuracy of Orientation Measurements using EBSD
}

\author{
K. Thomsen ${ }^{1}$, N.H. Schmidt ${ }^{1}$, A.Bewick ${ }^{2}$, K. Larsen ${ }^{2}$ and J. Goulden ${ }^{2}$ \\ 1. Oxford Instruments Consultant \\ 2. Oxford Instruments NanoAnalysis, Halifax Road, High Wycombe, HP12 3SE, UK
}

Routine EBSD analysis typically applies a 2D Hough transform technique. This requires collection and background correction of the Kikuchi pattern. A Hough transform is applied to highlight and locate the Kikuchi bands. These bands are revealed as local peaks in the resulting Hough space and their corresponding maxima are found automatically, locating the position and direction of the Kikuchi bands and the corresponding diffraction planes. In this primary band detection routine each maximum is represented by a (rho, theta) position in Hough space. In the subsequent indexing the interplanar angles are matched against a database of known phases, and the phase and orientation of the crystal is determined.

Precision and accuracy of this primary band detection is essentially limited by the precision of identifying and locating the Kikuchi bands, which is in turn limited by the Hough transform. Therefore, the precision in the orientation measurement can be improved by increasing the resolution of the Hough transform and the resulting Hough space; however this results in a significant increase in calculation time. In addition, the Hough transform assumes that the Kikuchi bands are straight lines, whereas in reality the Kikuchi bands in a pattern have varying widths and curved (hyperbolic) edges. Therefore the conventional 2D Hough transform method introduces a systematic error which cannot be overcome through increased Hough space resolution.

An alternative method [1] describes modifying the Hough transform so that it identifies hyperbolic curves rather than straight lines. This modified Hough transform applies a 3D space where the third dimension is given by the hyperbola variable. This method eliminates the systematic error of the conventional Hough, but it involves a large three dimensional calculation which makes it computationally slow.

This paper will present a new analysis routine for EBSPs, which improves the accuracy of band detection and is achievable in real time applications. The method uses the primary band detection as its foundation, and then applies an iterative secondary band detection to improve upon the accuracy of the primary band detection.

This accurate determination of band positions enables the calculation of a more accurate orientation matrix and thus improves general EBSD analysis, e.g. ability to distinguish between two phases with a very close crystal structure; or in characterizing low angle boundaries. In addition, this refinement will deliver an accurate solution when the primary band detection is impaired by the presence of excess and deficit lines.

The mean angular deviation (MAD) is a measure of the fit between the Kikuchi bands in the measured pattern and the solution. It can be used as figure of merit for the accuracy of band detection and therefore also for phase identification. Many applications of EBSD consider misorientation which is also related to the accuracy and precision of band detection. 
As in previous work [2] a single crystal silicon is used to characterize orientation precision; this removes complications resulting from the sample and simplifies analysis of different band detection and calibration methods. With a single crystal, the orientation should be constant throughout the field of view so differences in orientation from point to point are due to variability in the measurement.

As examples of the improvement offered by the new band detection, the same set of EBSPs from a single map have been processed using both the conventional Hough-based method (in blue); and the new refinement method (in red). Figure 1 is a plot of MAD distribution. Figure 2 is a plot of local misorientation distribution (local misorientation is a calculation of the average misorientation between each pixel and its nearest neighbors).

It is clear that by applying the secondary refinement both the local misorientation measurements and the MADs are significantly improved. These improvements indicate a more accurate fit between pattern and solution (MAD) which should yield benefits in ability to distinguish between phases and misorientation precision.

Further experimental work will be undertaken to demonstrate the benefit of this method across different EBSD applications.

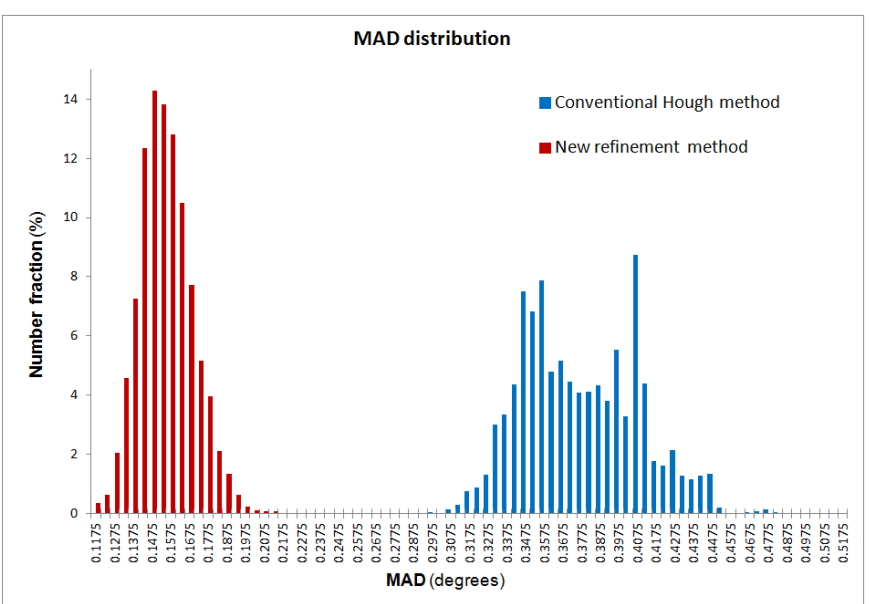

Figure 1 MAD distribution

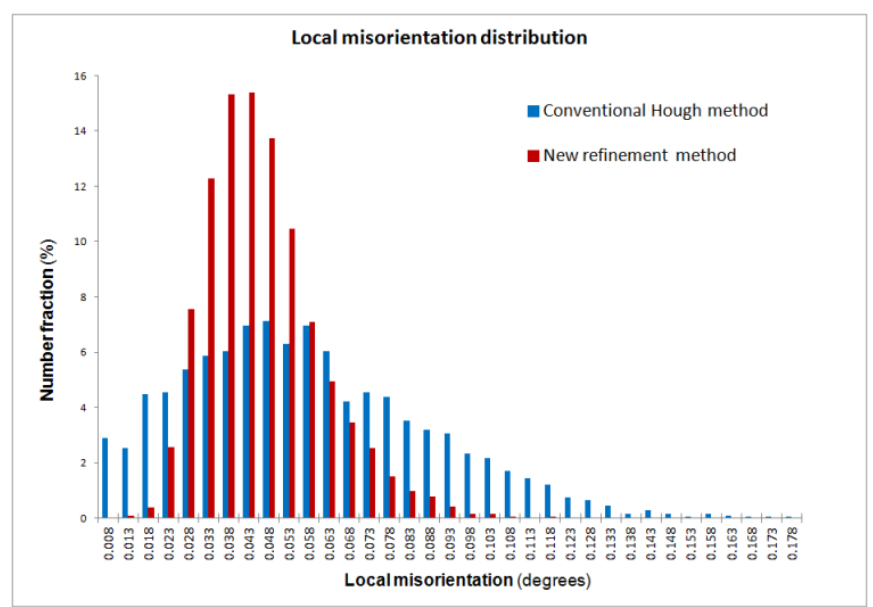

Figure 2 Local misorientation distribution

\section{References:}

[1] C. Maurice and R. Fortunier, "A 3D Hough transform for indexing EBSD and Kossel patterns", Journal of Microscopy, vol. 230, 2008, pp. 520-529

[2] S. Wright, M. Nowell, J. Basinger, "Precision of EBSD based Orientation Measurements" Microsc. Microanal. 17 (Suppl 2), 2011 